\title{
NOVEL PYRAZOLE-CENTERED DERIVATIVES HAVING MONO/DI CHIRAL CENTERED GROUP AS ORGANOCATALYST FOR HENRY REACTION
}

\author{
Adnan Çetin ${ }^{1, *}$, Ishak Bildirici², Selçuk Gümüş̧,** \\ ${ }^{1}$ Mus Alparslan University, Faculty of Education, Department of Sciences, Muş, Turkey \\ ${ }^{2}$ Van Yuzuncu Yil University, Faculty of Pharmacy, Department of Pharmaceutical Chemistry, Van, Turkey \\ ${ }^{3}$ Van Yuzuncu Yil University, Science Faculty, Department of Chemistry, Van, Turkey \\ a.cetin@alparslan.edu.tr, gumuss@gmail.com
}

\begin{abstract}
The chiral substituted pyrazole-3-carboxamides (4a-c), pyrazole-3-carboxylates (5a-c), pyrazole-3thioureides (7a-c) and pyrazole-3,4-dicarboxamides (10a-c) were prepared via the pyrazolo-3-chlorocarbonyl $\mathbf{2}$, pyrazolo-3,4-dicarboxy methyl ester $\mathbf{3}$ with pyrazole-3-isothiocyanate $\mathbf{6}$ with different $(R)$-chiral amino alcohols. All of the synthesized chiral compounds binding a pyrazole skeleton were investigated as organocatalysts for asymmetric aldol reactions between nitromethane and $p$-nitrobenzaldehyde in the presence of $\mathrm{CuCl}$. Enantiomeric excesses and the reaction yields were found to be appropriate values. Furthermore, the best organocatalyst applied in this study was identified after careful optimization of conditions. Lastly, all of the novel compounds were subjected to computational analysis at the B3LYP/6-31++G(d,p) level of theory to obtain information about their structural and electronic properties.
\end{abstract}

Keywords: asymmetric catalyst; chirality; chiral amino alcohol; Lewis acid; pyrazole

\section{НОВИ ПИРАЗОЛСКО ЦЕНТРИРАНИ ДЕРИВАТИ СО МОНО/ДИ ХИРАЛНО ЦЕНТРИРАНИ ГРУПИ КАКО ОРГАНОКАТАЛИЗАТОРИ ЗА ХЕНРИЕВА РЕАКЦИЈА}

\begin{abstract}
Беа подготвени хирално супституирани пиразол-3-карбоксамиди (4a-c), пиразол-3карбоксилати (5a-c), пиразол-3-тиоуреиди (7a-c) и пиразол-3,4-дикарбоксамиди (10a-c) преку пиразол-3-хлорокарбонил 2, пиразол-3,4-дикарбокси метил естер $\mathbf{3}$ со пиразоло-3-изотиоцијанат $\mathbf{6}$ со различни $(R)$-хирални аминоалкохоли. Сите синтетизирани хирални соединенија врзани за пиразолен скелет беа испитани како органокатализатори за асиметрични алдолни реакции меѓу нитрометан и $p$-нитробензалдехид во присуство на $\mathrm{CuCl}$. За погодни вредности се сметаа енантиомерниот вишок и приносите на реакциите. Покрај тоа, во ова истражување, по внимателно оптимизирање на условите, беше идентификуван најдобриот органокатализатор. Најпосле, сите нови соединенија беа подложени на компјутерска анализа на теориското ниво B3LYP/6$31++\mathrm{G}(\mathrm{d}, \mathrm{p})$ за да се добијат информации за нивната структура и електронски својства.
\end{abstract}

Клучни зборови: асиметричен катализатор; хиралност; хирални аминоалкохоли; Луисова киселина; пиразол

\section{INTRODUCTION}

The Henry (nitro aldol) reaction is known as the reaction between an in situ generated nitronate species and a carbonyl compound. It is one of the classical named carbon-carbon bond-forming reactions. This process provides a powerful and useful tool for the synthesis of valuable $\beta$-nitroalcohols. It is efficient and gives access to interesting and highly functionalized intermediates such as nitroalkenes, 1,2-amino alcohols, and $\alpha$-hydroxy carboxylic acids. Furthermore, the above-mentioned reaction and the resulting nitro aldol products play an important role both in synthetic organic chemistry and biological vitality [1]. This is related to the synthetic usefulness of its chiral centered products, as they can be 
easily converted to other useful synthetic compounds. Enantiomer rich nitro alkanol chiral compounds obtained by asymmetric catalysts have played key role in the synthesis of biologically active compounds. Also, chiral recognition is a selective interaction with one of the enantiomers of a chiral molecule or chiral receptor in living systems [2-3]. Chiral structures are important target molecules in chemistry since they have significant properties. They function as catalysts [4] and have biological activity [5] with implications in pharmaceutical [6], agricultural [7], and industrial [8] applications. In addition, the use of the enantiopure form of chiral drugs is now a basic requirement for every new chemical entity, and the development of new synthesis methods to obtain enantiopure compounds has become the most important goal for pharmaceutical chemistry. New methods developed for asymmetric synthesis are therefore becoming more important for synthetic chemists.

A wide variety of organo- and metal catalysts have been used for the Henry reaction. Among the most important asymmetric catalysts used for this reaction are chiral centered organocatalysts including carboxamide and thioureide functional groups [9].

On the other hand, since the beginning of this century, the substituted pyrazoles, which are an important class of heterocyclic compounds, have maintained continued interest in the literature because of the wide-spread research in this area, such as research on their use as catalysts. Additionally, the substituted pyrazoles reported in literature were prepared by processes such as asymmetric Michael reactions [10], enantioselective synthesis [11], selective complexation of metal ions [12], etc. [13-15]. Although the pyrazole compounds are very rare in natural products, they have very common synthetic applications. Pyrazole compounds have also been applied successfully in other fields such as in medicine [16], agriculture [17], industry [18], pharmaceutical chemistry [19], and optics [20]. For these reasons, they have quite an interesting research field in heterocyclic chemistry, and they have attracted interest for the synthesis of their derivatives and investigating new applications/methods.

Herein, we aimed to synthesize new different chiral substituted pyrazole-3-carboxamide, pyrazole-3-thioureide and pyrazole-3,4-dicarboxyamide derivatives via reactions of appropriate pyrazolo compounds with different $(R)$-chiral amino alcohols. Besides, the behavior of some these newly synthesized compounds as potent organocatalysts for nitro aldol reactions was investigated.
Carboxamide 4a-c and thioureide 7a-c derivatives were synthesized in our former article [21]. The other synthesized chiral compounds 5a-c and 10a-c have not been reported in the literature until now (Figure 1).

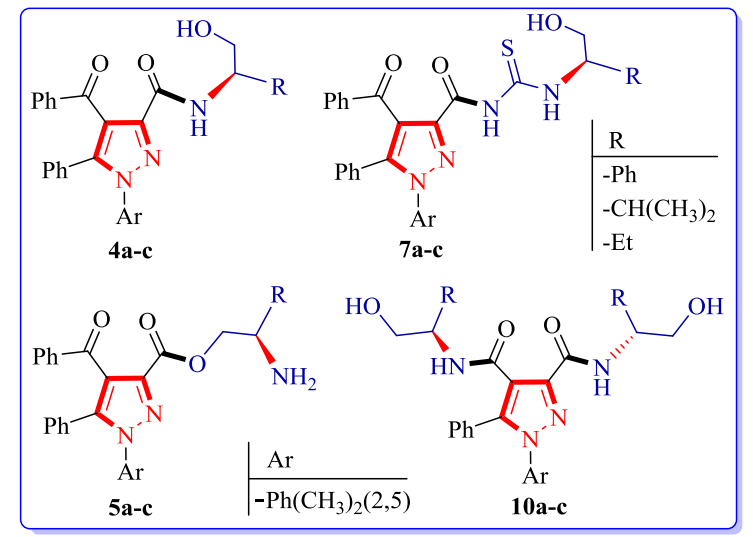

Fig. 1. Chiral substituted pyrazoles evaluated in this study

\section{EXPERIMENTAL}

\subsection{Materials and equipment}

All of the solvents and chemical reagents were purchased from commercial companies such as Merck and Sigma Aldrich. These materials were used without purification. ${ }^{1} \mathrm{H}(400 \mathrm{MHz})$ and ${ }^{13} \mathrm{C}$ $(100 \mathrm{MHz})$ NMR spectra were recorded on a Bruker DRX-400 high performance digital FTNMR spectrometer. NMR spectra were obtained in solutions of DMSO- $\mathrm{d}_{6}$ and $\mathrm{CDCl}_{3}$. Analytical TLC of all reactions was performed on Merck prepared plates. Infrared spectra were recorded on a Shimadzu IR-470 spectrophotometer. The elemental analyses were obtained with a Carlo-Erba Model 1108 apparatus. Optical rotations were taken on a Perkin Elmer 341 Model polarimeter. Refraction indices were measured by using an Atago Abbe refractometer. The mass spectra were measured on a Thermo Scientific TSQ-Quantum Access LC/MS spectrometer. Both pyrazole-3-carboxylic acid $\mathbf{1}$ (mp 202-204 ${ }^{\circ} \mathrm{C}$ ) and its acid chloride derivative $\mathbf{2}$ (mp $145{ }^{\circ} \mathrm{C}$ ) and the methyl ester $\mathbf{3}\left(\mathrm{mp} 180{ }^{\circ} \mathrm{C}\right.$ ) were our starting compounds, and they were synthesized by published procedures [22].

\subsection{Synthesis of chiral organocatalysts}

\section{General procedure for synthesis chiral pyrazole- 3-carboxamides (4a-c)}

Compounds 4a-c were synthesized by two different methods.

Method A: Compound 3 (0.410 g, $1 \mathrm{mmol})$ dissolved in methanol $(5 \mathrm{ml})$ was put to a reaction 
pot, and then a solution of chiral amino alcohols (1 mmol) (2-amino-2-phenylethanol, 2-amino-3methyl-1-butanol, 2-amino-1-butanol, respectively) in methanol $(5 \mathrm{ml})$ was added drop-wise to the solution at room temperature over 2 hours. After the addition, a solid product was precipitated, filtered, and washed with diethyl ether. The product obtained was purified by silica gel column chromatography ( $n$-hexane/ethyl acetate, 7:1).

Method B: An equimolar mixture of the acid chloride $2(0.414 \mathrm{~g}, 1 \mathrm{mmol})$ and a chiral amino alcohol (1 mmol) derivative was refluxed in xylene for 4 hours. After evaporation, the oily residue was treated with dry ether, and the crude product formed was purified by silica gel column chromatography ( $n$-hexane/ethyl acetate, 7:1). The first eluents from the column were pyrazole-3carboxamide derivatives (4a-c) as the main products (yield 65-80\%). The second compounds eluted were chiral pyrazole-3-carboxylate derivatives (5a-c) as by products (yield 10-15\%).

(R)-4-Benzoyl-1-(2,5-dimethylphenyl)- $N$-(2hydroxy-1-phenylethyl)-5-phenyl-1H-pyrazole3-carboxamide (4a)

Compound 4a was prepared according to general procedures existing in the literature [21]. Yield: 88 $\%$. $[\alpha]_{\mathrm{D}}^{23}+142.0^{\circ}(\mathrm{c}, 0,001, \mathrm{EtOH}), \mathrm{R}_{\mathrm{f}}=0.38, \mathrm{~m} . \mathrm{p}$ : 210-212 ${ }^{\circ} \mathrm{C}$. Anal. Calcd. for $\mathrm{C}_{33} \mathrm{H}_{29} \mathrm{~N}_{3} \mathrm{O}_{3}(M w=$ 515.22) C, 76.87; H, 5.67; N, 8.15 \%. Found: C, $76.71 ; \mathrm{H}, 5.59 ; \mathrm{N}, 8.30 \%$; IR $\left(v, \mathrm{~cm}^{-1}\right): 3275$, 3154, 3056, 2865, 1692, 1664, 1603, 1548, 1476, 1434, 1274; ${ }^{1} \mathrm{H}-\mathrm{NMR}$ (400 MHz, DMSO-d 6 , $\delta / \mathrm{ppm}): 8.42(d, 1 \mathrm{H}, J=9.4 \mathrm{~Hz}, \mathrm{NH}), 7.91-7.82$ $(m, 3 \mathrm{H}, \mathrm{Ar}-\mathrm{H}), 7.61-7.52(m, 5 \mathrm{H}, \mathrm{Ar}-\mathrm{H}), 7.24$ $7.15(m, 6 \mathrm{H}, \mathrm{Ar}-\mathrm{H}), 6.63-6.56(m, 4 \mathrm{H}, \mathrm{Ar}-\mathrm{H})$, 4.57-4.54 (m, 1H, CH-NH), 3.53-3.50 ( $m, 3 \mathrm{H}, \mathrm{OH}$ and $\left.\mathrm{CH}_{2} \mathrm{OH}\right), 2.17\left(s, 3 \mathrm{H}, \mathrm{CH}_{3}\right), 1.76(s, 3 \mathrm{H}$, $\left.\mathrm{CH}_{3}\right) ;{ }^{13} \mathrm{C}-\mathrm{NMR}\left(100 \mathrm{MHz}, \mathrm{DMSO}-\mathrm{d}_{6}, \delta / \mathrm{ppm}\right)$ : $192.4,166.2,145.3,143.6,141.3,139.5,137.6$, $136.2,134.5,133.6,132.3,121.2,129.7,128.1$, 123.6, 121.4, 116.5, 111.4, 63.2, 54.7, 21.6, 17.4. (+)ESI-HRMS m/z: calculated for $\left[\mathrm{C}_{33} \mathrm{H}_{29} \mathrm{~N}_{3} \mathrm{O}_{3}+\right.$ $\mathrm{H}^{+}$]: 516.2282; observed 516.2271. HPLC analysis: Chiral OD, $n$-hexane/isopropyl alcohol 85:15 (v:v), $0.5 \mathrm{ml} \mathrm{min}^{-1}, \lambda=254 \mathrm{~nm}, \mathrm{t}_{\mathrm{R}}$ (minor) $=15.49$ $\min , \mathrm{t}_{\mathrm{R}}($ major $)=18.25 \mathrm{~min}$.

(R)-4-Benzoyl-1-(2,5-dimethylphenyl) - $N$-(1hydroxy-3-methylbutan-2-yl)-5-phenyl-1Hpyrazole-3-carboxamide (4b)

Compound 4b was prepared according to general procedures existing in the literature [21]. Yield: 85 $\%$. $[\alpha]_{\mathrm{D}}^{23}-204.0^{\circ}(\mathrm{c}, 0,001, \mathrm{EtOH}), \mathrm{R}_{\mathrm{f}}=0.38$; m.p: 191-193 ${ }^{\circ} \mathrm{C}$. Anal. Calcd. for $\mathrm{C}_{30} \mathrm{H}_{31} \mathrm{~N}_{3} \mathrm{O}_{3}(M w=$ 481.24): C, 74.82; H, 6.49; N, $8.73 \%$. Found: C, 74.69; H, 6.61; N, $8.92 \%$. IR $\left(v, \mathrm{~cm}^{-1}\right): 3312$,
3168, 3040, 2818, 1696, 1668, 1576, 1482, 1401, 1370, 1315. ${ }^{1} \mathrm{H}-\mathrm{NMR} \quad\left(400 \mathrm{MHz}, \mathrm{DMSO}-\mathrm{d}_{6}\right.$, $\delta / \mathrm{ppm}): 8.35(d, 1 \mathrm{H}, J=9.2 \mathrm{~Hz}, \mathrm{NH}), 7.90-7.88$ ( $m, 2 \mathrm{H}$, Ar-H), 7.78-7.77 ( $m, 2 \mathrm{H}$, Ar-H), 7.26$7.16(m, 5 \mathrm{H}, \mathrm{Ar}-\mathrm{H}), 6.76-6.52(m, 4 \mathrm{H}, \mathrm{Ar}-\mathrm{H})$, 3.71-3.58 (m, 3H, $\mathrm{CH}-\mathrm{NH}$ and $\left.\mathrm{CH}_{2}-\mathrm{OH}\right), 2.50(\mathrm{~m}$, $2 \mathrm{H}, \mathrm{CH}$ and $\mathrm{OH}), 2.18-2.16\left(m, 6 \mathrm{H}, 2 \times \mathrm{CH}_{3}\right)$, 1.61-1.34 (m, 6H, $\left.2 \times \mathrm{CH}_{3}\right) ;{ }^{13} \mathrm{C}-\mathrm{NMR}(100 \mathrm{MHz}$, DMSO-d 6 , $\delta / p p m: 194.2,162.5,144.1,142.7$, $141.5,140.1,138.6,136.4,135.3,133.1,132.0$, $130.5,129.1,128.4,127.6122 .3,121.1,109.2$, $63.3, \quad 55.3, \quad 30.1, \quad 21.0, \quad 18.4, \quad 12.2 . \quad(+)$ ESIHRMS $m / z$ : calculated for $\left[\mathrm{C}_{30} \mathrm{H}_{31} \mathrm{~N}_{3} \mathrm{O}_{3}+\mathrm{H}^{+}\right]$: 482.2444; observed 482.2441. HPLC analysis: Chiral OD, $n$-hexane/isopropyl alcohol 85:15 (v:v), $0.5 \mathrm{ml} \mathrm{min}{ }^{-1}, \lambda=254 \mathrm{~nm}, \mathrm{t}_{\mathrm{R}}$ (minor) $=14.58 \mathrm{~min}$, $\mathrm{t}_{\mathrm{R}}($ major $)=17.25 \mathrm{~min}$.

(R)-4-Benzoyl-1-(2,5-dimethylphenyl)- $N$-(1hydroxybutan-2-yl)-5-phenyl-1H-pyrazole-3carboxamide (4c)

Compound $\mathbf{4 c}$ was prepared according to general procedures existing in the literature [21]. Yield: 85 $\% .[\alpha]_{\mathrm{D}}^{23}+186.3^{\circ}(\mathrm{c}, 0,001, \mathrm{EtOH}), \mathrm{R}_{\mathrm{f}}=0.39$, m.p: 199-201 ${ }^{\circ} \mathrm{C}$. Anal. Calcd. for $\mathrm{C}_{29} \mathrm{H}_{29} \mathrm{~N}_{3} \mathrm{O}_{3}(M w=$ 467.22): C, 74.50; H, 6.25; N, $8.99 \%$. Found: C, $74.71 ; \mathrm{H}, 6.42 ; \mathrm{N}, 8.73 \%$; IR $\left(v, \mathrm{~cm}^{-1}\right): 3362$, 3156, 3066, 1576, 1489, 1385, 1310; ${ }^{1} \mathrm{H}-\mathrm{NMR}$ (400 MHz, $\left.\mathrm{CDCl}_{3}, \delta / \mathrm{ppm}\right): 8.34(d, 1 \mathrm{H}, J=9.2$ $\mathrm{Hz}, \mathrm{NH}), 7.88-7.74(m, 2 \mathrm{H}, \mathrm{Ar}-\mathrm{H}), 7.52-7.45(m$, 5H, Ar-H), 7.25-7.14 ( $m, 5 \mathrm{H}$, Ar-H), 6.78-6.76 ( $m, 1 \mathrm{H}, \mathrm{Ar}-\mathrm{H}), 3.84-3.80\left(m, 2 \mathrm{H}, \mathrm{CH}_{2}-\mathrm{OH}\right), 3.52-$ $3.50(\mathrm{~m}, 1 \mathrm{H}, \mathrm{CH}-\mathrm{NH}), 2.68(s, 1 \mathrm{H}, \mathrm{OH}), 2.13(s$, $\left.3 \mathrm{H}, \mathrm{CH}_{3}\right), 1.87\left(s, 3 \mathrm{H}, \mathrm{CH}_{3}\right), 1.62\left(m, 2 \mathrm{H}, \mathrm{CH}_{2}-\right.$ $\left.\mathrm{CH}_{3}\right), 1.35$ (t, $\left.3 \mathrm{H}, J=7.1 \mathrm{~Hz}, \mathrm{CH}_{3}\right) ;{ }^{13} \mathrm{C}-\mathrm{NMR}(100$ $\left.\mathrm{MHz}, \mathrm{CDCl}_{3}, \delta / \mathrm{ppm}\right)$ : 198.3, 164.7, 146.3, 143.2, $141.5,134.1,133.9,132.9,131.6,130.8,130.0$, $129.5,129.1,128.1,126.3,125.4,121.1,108.2$, 58.6, 55.3, 30.1, 22.1, 17.3, 14.6. (+)ESIHRMS $m / z$ : calculated for $\left[\mathrm{C}_{29} \mathrm{H}_{29} \mathrm{~N}_{3} \mathrm{O}_{3}+\mathrm{H}^{+}\right]$: 468.2282; observed 468.2287. HPLC analysis: Chiral OD, $n$-hexane/isopropyl alcohol 85:15 (v:v), $0.5 \mathrm{ml} \mathrm{min}{ }^{-1}, \lambda=254 \mathrm{~nm}, \mathrm{t}_{\mathrm{R}}$ (minor) $=15.36 \mathrm{~min}$, $\mathrm{t}_{\mathrm{R}}($ major $)=18.21 \mathrm{~min}$.

\section{General procedure for synthesis of chiral pyrazole-3-carboxylates (5a-c)}

Pyrazole-3-carboxylate (5a-c) derivatives were obtained as given above in method B which introduces the synthesis of $\mathbf{4 a - c}$ compounds.

(R)-2-Amino-2-phenylethyl4-benzoyl-1-(2,5dimethylphenyl)-5-phenyl-1H-pyrazole-3carboxylate (5a)

Compound 5a was prepared according to method B $(0.09 \mathrm{~g}, 12 \%) .[\alpha]^{23} \mathrm{D}+160.1^{\circ}(\mathrm{c}, 0,001 \mathrm{EtOH})$; mp: $172-174{ }^{\circ} \mathrm{C} ; \mathrm{R}_{\mathrm{f}}=0.36$, IR $\left(v, \mathrm{~cm}^{-1}\right): 3125$, 
3064, 1710, 1596, 1561, 1484, 1442, 1374, 1315; ${ }^{1} \mathrm{H}-\mathrm{NMR}\left(400 \mathrm{MHz}, \mathrm{CDCl}_{3}\right) \delta$ (ppm): 8.32-8.31 ( $m, 2 \mathrm{H}$, Ar-H) $7.72-7.63(m, 4 \mathrm{H}$, Ar-H), 7.24$7.18(m, 6 \mathrm{H}, \mathrm{Ar}-\mathrm{H}), 6.71-6.69(m, 4 \mathrm{H}, \mathrm{Ar}-\mathrm{H})$, 6.32-6.31 (m, 2H, Ar-H), 4.43-4.42 ( $m, 2 \mathrm{H}, \mathrm{CH}_{2-}$ O), 3.42-3.41 (m, 1H, CH-NH), $2.28\left(s, 3 \mathrm{H}, \mathrm{CH}_{3}\right)$, $1.90\left(m, 2 \mathrm{H}, \mathrm{NH}_{2}\right), 1.53\left(s, 3 \mathrm{H}, \mathrm{CH}_{3}\right) ;{ }^{13} \mathrm{C}-\mathrm{NMR}$ $\left(100 \mathrm{MHz}, \mathrm{CDCl}_{3}\right) \delta(\mathrm{ppm}): 191.4,163.6,152.1$, $146.3,142.1,137.1,133.1,132.7,131.8,130.6$, $130.1,129.6,129.2,128.8,128.7,128.5,128.4$, 121.3, 108.4, 54.6, 38.2, 22.3, 17.1. Anal. Calcd. for $\mathrm{C}_{33} \mathrm{H}_{29} \mathrm{~N}_{3} \mathrm{O}_{3}: \mathrm{C}, 76.87 ; \mathrm{H}, 5.67 ; \mathrm{N}, 8.15$. Found: C, 76.21; H, 5.69; N, 8.09. (+)ESI-HRMS $m / z$ : calculated for $\left[\mathrm{C}_{33} \mathrm{H}_{29} \mathrm{~N}_{3} \mathrm{O}_{3}+\mathrm{H}^{+}\right]$: 516.2282; observed 516.2275. HPLC analysis: Chiral OD, $n$ hexane/isopropyl alcohol 85:15 (v:v), $0.5 \mathrm{ml} \mathrm{min}^{-1}$, $\lambda=254 \mathrm{~nm}, \mathrm{t}_{\mathrm{R}}($ major $)=15.23$ min, $\mathrm{t}_{\mathrm{R}}($ minor $)=$ $17.26 \mathrm{~min}$.

(R)-2-Amino-3-methylbutyl4-benzoyl-1-(2,5dimethylphenyl)-5-phenyl-1H-pyrazole-3carboxylate (5b)

Compound $\mathbf{5 b}$ was prepared according to method B $(0.08$ g, $15 \%)$. [ $\alpha]^{23}{ }^{2}+114.8^{\circ}(\mathrm{c}, 0,001 \mathrm{EtOH})$; mp: $181-184{ }^{\circ} \mathrm{C} ; \mathrm{R}_{\mathrm{f}}=0.36, \mathrm{IR}\left(\mathrm{v}, \mathrm{cm}^{-1}\right): 3142$, 3060, 1708, 1598, 1556, 1482, 1444, 1365, 1321; ${ }^{1} \mathrm{H}-\mathrm{NMR}\left(400 \mathrm{MHz}, \mathrm{CDCl}_{3}\right) \delta$ (ppm): 8.12-8.10 $(m, 1 \mathrm{H}, \mathrm{Ar}-\mathrm{H}), 7.95-7.81(m, 4 \mathrm{H}, \mathrm{Ar}-\mathrm{H}), 7.51-$ $7.47(m, 4 \mathrm{H}, \mathrm{Ar}-\mathrm{H}), 7.26-7.23(m, 2 \mathrm{H}, \mathrm{Ar}-\mathrm{H})$, 7.10-7.08 ( $m, 1 \mathrm{H}$, Ar-H $), 6.66-6.64(m, 1 \mathrm{H}, \mathrm{Ar}-$ $\mathrm{H}), 3.73-3.70\left(\mathrm{~m}, 2 \mathrm{H}, \mathrm{CH}_{2}-\mathrm{O}\right), 2.82-2.81(\mathrm{~m}, 1 \mathrm{H}$, $C H-\mathrm{NH}), 2.35\left(s, 3 \mathrm{H}, \mathrm{CH}_{3}\right), 1.77\left(s, 3 \mathrm{H}, \mathrm{CH}_{3}\right)$, $1.58\left(\mathrm{~m}, 3 \mathrm{H}, \mathrm{CH}\right.$ and $\left.\mathrm{NH}_{2}\right), 1.20\left(s, 6 \mathrm{H}, 2 \mathrm{xCH}_{3}\right)$; ${ }^{13} \mathrm{C}-\mathrm{NMR}\left(100 \mathrm{MHz}, \mathrm{CDCl}_{3}\right) \delta(\mathrm{ppm}): 193.2$, $160.0,145.1,141.3,138.2,136.4,133.2,130.5$, $130.4,129.6,129.5,129.4,128.7,128.5,128.3$, $128.1,122.1,109.7,79.2,42.2,27.5,20.2,16.4$, 12.2. Anal. Calcd. for $\mathrm{C}_{30} \mathrm{H}_{31} \mathrm{~N}_{3} \mathrm{O}_{3}: \mathrm{C}, 74.82 ; \mathrm{H}$, 6.49; N, 8.73. Found: C, 74.25; H, 6.51; N, 8.69. (+)ESI-HRMS m/z: calculated for $\left[\mathrm{C}_{30} \mathrm{H}_{31} \mathrm{~N}_{3} \mathrm{O}_{3}+\right.$ $\mathrm{H}^{+}$]: 482.2444; observed 482.2438. HPLC analysis: Chiral OD, $n$-hexane/isopropyl alcohol $85: 15(\mathrm{v}: \mathrm{v}), 0.5 \mathrm{ml} \mathrm{min}^{-1}, \lambda=254 \mathrm{~nm}, \mathrm{t}_{\mathrm{R}}$ (major) $=$ $15.43 \mathrm{~min}, \mathrm{t}_{\mathrm{R}}($ minor $)=17.26 \mathrm{~min}$.

(R)-2-Aminobutyl4-benzoyl-1-(2,5-dimethylphenyl)-5-phenyl-1 $\mathrm{H}$-pyrazole-3-carboxylate (5c) Compound $\mathbf{5 c}$ was prepared according to method B $(0.08 \mathrm{~g}, 15 \%) .[\alpha]^{23} \mathrm{D}+114.8^{\circ}$ (c, 0,001 EtOH); mp: $181-184{ }^{\circ} \mathrm{C} ; \mathrm{R}_{\mathrm{f}}=0.36, \mathrm{IR}\left(v, \mathrm{~cm}^{-1}\right): 3135$, 3064, 1716, 1596, 1552, 1492, 1447, 1352, 1317; ${ }^{1} \mathrm{H}-\mathrm{NMR}\left(400 \mathrm{MHz}, \mathrm{CDCl}_{3}\right) \delta$ (ppm): 8.05-7.97 $(m, 1 \mathrm{H}, \mathrm{Ar}-\mathrm{H}), 7.92-7.87(m, 4 \mathrm{H}$, Ar- $\mathrm{H}), 7.68$ $7.64(m, 4 \mathrm{H}$, Ar-H), 7.27-7.24 (m, 2H, Ar-H), 7.08-7.04 (m, 1H, Ar-H), 6.48-6.46 (m, 1H, Ar$\mathrm{H}), 3.72-3.70\left(\mathrm{~m}, 2 \mathrm{H}, \mathrm{CH}_{2}-\mathrm{O}\right), 2.82-2.80(\mathrm{~m}, 1 \mathrm{H}$,
$\mathrm{CH}-\mathrm{N}), 2.20-1.80\left(\mathrm{~m}, 8 \mathrm{H}, \mathrm{CH}_{2}-\mathrm{CH}_{3}\right.$ and $\left.2 \times \mathrm{CH}_{3}\right)$, $1.48\left(b, 2 \mathrm{H}, \mathrm{NH}_{2}\right), 1.16\left(t, J=7.1 \mathrm{~Hz}, 3 \mathrm{H}, \mathrm{CH}_{3}\right)$; ${ }^{13} \mathrm{C}-\mathrm{NMR}\left(100 \mathrm{MHz}, \mathrm{CDCl}_{3}\right) \delta$ (ppm): 194.6, $158.3,142.1,138.0,136.4,133.0,131.9,131.0$, $130.7,130.3,129.7,129.6,129.5,129.4,128.8$, 128.1, 122.7, 121.3, 109.2, 81.6, 42.7, 24.6, 20.9, 17.3, 11.0. Anal. Calcd. for $\mathrm{C}_{30} \mathrm{H}_{31} \mathrm{~N}_{3} \mathrm{O}_{3}$ : C, 74.82; H, 6.49; N, 8.73. Found: C, 74.81; H, 6.47; N, 8.74. (+)ESI-HRMS m/z: calculated for $\left[\mathrm{C}_{29} \mathrm{H}_{29} \mathrm{~N}_{3} \mathrm{O}_{3}+\right.$ $\mathrm{H}^{+}$]: 468.2282; observed 468.2287. HPLC analysis: Chiral OD, $n$-hexane/isopropyl alcohol 85:15 (v:v), $0.5 \mathrm{ml} \mathrm{min}^{-1}, \lambda=254 \mathrm{~nm}, \mathrm{t}_{\mathrm{R}}$ (major) $=15.21 \mathrm{~min}$, $\mathrm{t}_{\mathrm{R}}($ minor $)=17.49 \mathrm{~min}$.

\section{4-Benzoyl-1-(2,5-dimethylphenyl)-5-phenyl-1 $H$ - pyrazole-3-carbonyl-isothiocyanate (6)}

To synthesize compound $\mathbf{6}$, a procedure existing in the literature was followed [21, 22]. The yield of $\mathbf{6}$ was $60 \%$; mp $159-160.5^{\circ} \mathrm{C}$.

Synthesis of chiral pyrazole-3-thioureides (7a-c) In general, pyrazole-3-thioureide derivatives (7a-c) were obtained according to our previous study [21].

(R)-1-(4-Benzoyl-1-(2,5-dimethylphenyl)-5phenyl-1H-pyrazole-3-carbonyl)-3-(2-hydroxy1-phenylethyl)thiourea (7a)

Compound 7a was prepared according to the literature $(0.36 \mathrm{~g}, 61 \%)$ [21]. Yield: $61 \% .[\alpha]_{\mathrm{D}}{ }^{23}+192.3^{\circ}$ (c, 0,005, EtOH), $\mathrm{R}_{\mathrm{f}}=0.37$, m.p: $231-233^{\circ} \mathrm{C}$. Anal. Calcd. for $\mathrm{C}_{34} \mathrm{H}_{30} \mathrm{~N}_{4} \mathrm{O}_{3} \mathrm{~S}(M w=574.20)$ : $\mathrm{C}, 71.06 ; \mathrm{H}$, 5.26; N, $9.75 \%$. Found: C, 71.23; H, 5.34; N, 9.79 $\%$; IR $\left(v, \mathrm{~cm}^{-1}\right): 3355,3212,3054,2925,1712,1686$, 1674, 1572, 1494, 1442, 1323. 'H-NMR (400 MHz, DMSO-d $\left._{6}, \delta / \mathrm{ppm}\right): 8.30-8.28(m, 1 \mathrm{H}, \mathrm{NH}), 8.21-$ 7.04 ( $m, 16 \mathrm{H}, \mathrm{Ar}-\mathrm{H}), 6.58-6.56(\mathrm{~m}, 2 \mathrm{H}, \mathrm{Ar}-\mathrm{H}), 3.96-$ $3.94\left(m, 2 \mathrm{H}, \mathrm{CH}_{2}-\mathrm{OH}\right), 3.14-3.12(\mathrm{~m}, 1 \mathrm{H}, \mathrm{CH}-\mathrm{NH})$, 2.40-2.17 ( $m, 4 \mathrm{H}, \mathrm{OH}$ and $\left.\mathrm{CH}_{3}\right), 1.18\left(b s, 3 \mathrm{H}, \mathrm{CH}_{3}\right)$; ${ }^{13} \mathrm{C}-\mathrm{NMR}$ (100 MHz, DMSO-d 6 , $\left.\delta / \mathrm{ppm}\right): 198.3$, $188.6,166.7,143.1,141.2,136.7,135.4,132.6$, $132.2,131.5,131.1,130.4,130.2,130.0,129.9$, $129.8,129.6,129.3,129.2,129.1,128.7,128.5$, $128.1,127.2,122.5,109.1,64.2,50.3,21.4,16.8$. (+)ESI-HRMS m/z: calculated for $\left[\mathrm{C}_{34} \mathrm{H}_{30} \mathrm{~N}_{4} \mathrm{O}_{3} \mathrm{~S}+\right.$ $\mathrm{H}^{+}$]: 575.2117; observed 575.2111. HPLC analysis: Chiral OD, $n$-hexane/ isopropyl alcohol 85:15 (v:v), $0.5 \mathrm{ml} \mathrm{min}{ }^{-1}, \lambda=254 \mathrm{~nm}, \mathrm{t}_{\mathrm{R}}$ (minor) $=16.41 \mathrm{~min}, \mathrm{t}_{\mathrm{R}}$ $($ major $)=18.53 \mathrm{~min}$.

\section{(R)-1-(4-Benzoyl-1-(2,5-dimethylphenyl)-5- phenyl-1H-pyrazole-3-carbonyl)-3-(1-hydroxy- 3-methylbutan-2-yl)thiourea (7b) \\ Compound $\mathbf{7 b}$ was prepared according to the lit- erature $(0.3 \mathrm{~g}, 56 \%)$ [21]. Yield: $56 \%$. $[\alpha]_{\mathrm{D}}{ }^{23}$ $+125.4^{\circ}$ (c, 0,005, EtOH), $\mathrm{R}_{\mathrm{f}}=0.37$, m.p: 207-209 ${ }^{\circ} \mathrm{C}$. Anal. Calcd. for $\mathrm{C}_{31} \mathrm{H}_{32} \mathrm{~N}_{4} \mathrm{O}_{3} \mathrm{~S}(M w=540.22)$ :}


C, 68.87; H, 5.97; N, $10.36 \%$. Found: C, 68.95; H, $5.89 ; \mathrm{N}, 10.42 \%$; IR $\left(v, \mathrm{~cm}^{-1}\right): 3365,3151,3062$, 2957, 1704, 1686, 1671, 1568, 1498, 1444, 1321. ${ }^{1} \mathrm{H}-\mathrm{NMR}$ (400 MHz, DMSO-d 6 , $\left.\delta / \mathrm{ppm}\right): 8.13-8.11$ $(m, 1 \mathrm{H}, \mathrm{NH}), 8.05-7.20(m, 12 \mathrm{H}, \mathrm{Ar}-\mathrm{H}), 6.65-6.62$ $(m, 1 \mathrm{H}, \mathrm{Ar}-\mathrm{H}), 3.95-3.92\left(m, 2 \mathrm{H}, \mathrm{CH}_{2}-\mathrm{OH}\right), 2.89$ $2.87(m, 1 \mathrm{H}, \mathrm{CH}-\mathrm{NH}), 2.41(b s, 1 \mathrm{H}, \mathrm{OH}), 2.21(b s$, $\left.3 \mathrm{H}, \mathrm{CH}_{3}\right), 1.96-1.94(m, 1 \mathrm{H}, \mathrm{CH}-), 1.79(b s, 3 \mathrm{H}$, $\left.\mathrm{CH}_{3}\right), 1.66-1.62\left(\mathrm{~m}, 3 \mathrm{H}, \mathrm{CH}_{3}\right), 1.18\left(\mathrm{bs}, 3 \mathrm{H}, \mathrm{CH}_{3}\right)$; ${ }^{13} \mathrm{C}-\mathrm{NMR}$ (100 MHz, DMSO-d 6 , $\left.\delta / \mathrm{ppm}\right): 196.8$, $180.3,165.9,144.7,142.3,141.8,137.5,135.2$, $134.4,133.2,132.1,131.7,129.8,129.1,128.6$, $127.5,126.3,125.4,124.2,121.7,108.2,64.1$, 59.7, 32.1, 21.4, 18.4, 17.2. (+)ESI-HRMS m/z: calculated for $\left[\mathrm{C}_{31} \mathrm{H}_{32} \mathrm{~N}_{4} \mathrm{O}_{3} \mathrm{~S}+\mathrm{H}^{+}\right]:$541.2273; observed 541.2269. HPLC analysis: Chiral OD, $n$ hexane/isopropyl alcohol 85:15 (v:v), $0.5 \mathrm{ml} \mathrm{min}^{-1}, \lambda$ $=254 \mathrm{~nm}, \mathrm{t}_{\mathrm{R}}($ minor $)=14.51 \mathrm{~min}, \mathrm{t}_{\mathrm{R}}($ major $)=$ $17.26 \mathrm{~min}$.

(R)-1-(4-Benzoyl-1-(2,5-dimethylphenyl)-5phenyl-1H-pyrazole-3-carbonyl)-3-(1-hydroxy butan-2-yl)thiourea (7c)

Compound 7c was prepared according to the literature $(0.26 \mathrm{~g}, 50 \%)$ [21]. Yield: $50 \%$. $[\alpha]_{\mathrm{D}}^{23}$ $+153.2^{\circ}$ (c, 0,005, EtOH), $\mathrm{R}_{\mathrm{f}}=0.37$, m.p: $215-217$ ${ }^{\circ} \mathrm{C}$. Anal. Calcd. for $\mathrm{C}_{30} \mathrm{H}_{32} \mathrm{~N}_{4} \mathrm{O}_{3} \mathrm{~S}(M w=512.19)$ : C, 67.95; H, 5.51; N, $10.93 \%$. Found: C, 68.56; H, 5.91; N, $10.51 \%$; IR $\left(v, \mathrm{~cm}^{-1}\right): 3435,3165,3058$, 2895, 1711, 1685, 1667, 1572, 1498, 1442, 1323. ${ }^{1} \mathrm{H}-\mathrm{NMR}$ (400 MHz, DMSO-d 6 , $\delta / \mathrm{ppm}$ ): 8.67 (bs, $1 \mathrm{H}, \mathrm{NH}), 8.23-6.97(m, 13 \mathrm{H}, \mathrm{NH}$ and $\mathrm{Ar}-\mathrm{H}), 6.84-$ $6.82(m, 1 \mathrm{H}, \mathrm{Ar}-\mathrm{H}), 3.76-3.71\left(\mathrm{~m}, 2 \mathrm{H}, \mathrm{CH}_{2}-\mathrm{OH}\right)$, 2.82-2.80 ( $m, 1 \mathrm{H}, C H-\mathrm{NH}), 2.42-2.25(m, 4 \mathrm{H}, \mathrm{OH}$ and $\left.\mathrm{CH}_{3}\right), 2.13$ (bs, 3H, $\left.\mathrm{CH}_{3}\right), 1.66-1.63(\mathrm{~m}, 2 \mathrm{H}$, $\left.\mathrm{CH}_{2}-\mathrm{CH}_{3}\right), 1.10-1.06\left(\mathrm{~m}, 3 \mathrm{H}, \mathrm{CH}_{3}\right) ;{ }^{13} \mathrm{C}-\mathrm{NMR}(100$ MHz, DMSO- $\left.\mathrm{d}_{6}, \delta / \mathrm{ppm}\right): 194.5,181.0,167.1$, $144.4,141.6,139.5,137.2,136.7,135.3,131.5$, $130.3,129.9,129.4,129.1,128.8,128.6,128.4$, 128.0, 127.5, 124.3, 108.8, 62.3, 55.7, 27.3, 22.6, 17.1, 10.6. (+)ESI-HRMS $\mathrm{m} / \mathrm{z}$ : calculated for $\left[\mathrm{C}_{30} \mathrm{H}_{30} \mathrm{~N}_{4} \mathrm{O}_{3} \mathrm{~S}+\mathrm{H}^{+}\right]$: 527.2117; observed 527.2110. HPLC analysis: Chiral OD, $n$-hexane/isopropyl alcohol 85:15 (v:v), $0.5 \mathrm{ml} \mathrm{min}^{-1}, \lambda=254 \mathrm{~nm}, \mathrm{t}_{\mathrm{R}}\left(\mathrm{mi}^{-}\right.$ nor $)=16.27 \mathrm{~min}, \mathrm{t}_{\mathrm{R}}($ major $)=17.26 \mathrm{~min}$.

\section{1-(2,5-Dimethylphenyl)-4-(ethoxycarbonyl)-5-} phenyl-1H-pyrazole-3-carboxylic acid (8)

Compound 8 was obtained according to our previous study [22]. The yield of 8 was $65 \%$; mp $202^{\circ} \mathrm{C}$.

\section{Diethyl1-(2,5-dimethylphenyl)-5-phenyl-1H- pyrazole-3,4-dicarboxylate (9)}

To synthesize compound $\mathbf{9}$, a literature procedure was followed [22]. The yield of 9 was $73 \%$; mp $165^{\circ} \mathrm{C}$.
General procedure for synthesis of chiral pyrazole-3,4-dicarboxamides (10a-c)

Compound 9 (0.196 g $0.5 \mathrm{mmol})$ dissolved in ethyl alcohol $(5 \mathrm{ml})$ was added dropwise to the appropriate chiral amino alcohol $(1 \mathrm{mmol})$ (2-amino-2phenylethanol, 2-amino-3-methyl-1-butanol, 2amino-1-butanol, respectively) in ethyl alcohol (5 $\mathrm{ml})$ at room temperature. This mixture was stirred at room temperature for 2 hours. A solid residue was formed in the reaction mixture. The solid product was precipitated, filtered, and dried; the product was purified by crystallization from ethanol.

1-(2,5-Dimethylphenyl)- $N^{3}, N^{4}$-bis(R)-2-hydroxy1-phenylethyl)-5-phenyl-1H-pyrazole-3,4dicarboxamide (10a)

Compound 10a was prepared according to the general procedure $(0.29 \mathrm{~g}, 86 \%)$. Mp: $252-254{ }^{\circ} \mathrm{C}$; IR $\left(v, \mathrm{~cm}^{-1}\right): 3356,3125,3066,2910,1664,1658$, 1584, 1492, 1444, 1320; ${ }^{1} \mathrm{H}-\mathrm{NMR}$ (400 MHz, $\left.\mathrm{CDCl}_{3}\right) \delta$ (ppm): 9.68-9.67 ( $\left.m, 2 \mathrm{H}, \mathrm{NH}\right), 8.09-$ $8.04(m, 3 \mathrm{H}, \mathrm{Ar}-\mathrm{H}), 7.65-7.59(m, 4 \mathrm{H}, \mathrm{Ar}-\mathrm{H})$, 7.49-7.47 (m, 4H, Ar-H), 7.26-7.25 ( $m, 2 \mathrm{H}, \mathrm{Ar}-$ $\mathrm{H}), 6.84-6.83(m, 3 \mathrm{H}, \mathrm{Ar}-\mathrm{H}), 6.59-6.52(m, 2 \mathrm{H}$, Ar-H), 4.89-4.86 ( $m, 1 \mathrm{H}, \mathrm{CH}-\mathrm{NH}), 4.0(m, 2 \mathrm{H}$, $\mathrm{OH}), 3.53-3.50\left(\mathrm{~m}, 2 \mathrm{H}, \mathrm{CH}_{2}-\mathrm{OH}\right), 3.19-3.18(\mathrm{~m}$, $\left.2 \mathrm{H}, \mathrm{CH}_{2} \mathrm{OH}\right), 2.20\left(\mathrm{~s}, 3 \mathrm{H}, \mathrm{CH}_{3}\right), 1.50(\mathrm{~s}, 3 \mathrm{H}$, $\left.\mathrm{CH}_{3}\right) ;{ }^{13} \mathrm{C}-\mathrm{NMR}\left(100 \mathrm{MHz}, \mathrm{CDCl}_{3}\right) \delta$ (ppm): $166.3,164.1,145.2,141.1,138.6,136.0,135.2$, $133.8,132.5,131.5,130.8,129.9,129.6,129.5$, $129.4,129.3,129.1,128.6,127.1,121.5,112.4$, 53.5, 46.1, 22.3, 17.4. Anal. Calcd. for $\mathrm{C}_{35} \mathrm{H}_{34} \mathrm{~N}_{4} \mathrm{O}_{4}$ : C, 73.15; H, 5.96; N, 9.75. Found: C, 72.49; H, 5.95; N, 9.79. (+)ESI-HRMS $m / z$ : calculated for $\left[\mathrm{C}_{33} \mathrm{H}_{29} \mathrm{~N}_{3} \mathrm{O}_{3}+\mathrm{H}^{+}\right]$: 575.2653; observed 575.2648. HPLC analysis: Chiral OD, $n$ hexane/isopropyl alcohol 85:15 (v:v), $0.5 \mathrm{ml} \mathrm{min}^{-1}$, $\lambda=254 \mathrm{~nm}, \mathrm{t}_{\mathrm{R}}($ minor $)=14.52 \mathrm{~min}, \mathrm{t}_{\mathrm{R}}($ major $)=$ $17.19 \mathrm{~min}$.

1-(2,5-Dimethylphenyl)- $N^{3}, N^{4}$-bis $(R)$-1-hydroxy3-methylbutan-2-yl)-5-phenyl-1H-pyrazole-3,4dicarboxamide (10b)

Compound 10b was prepared according to general procedure $(0.26 \mathrm{~g}, 88 \%)$. Mp: $246-248{ }^{\circ} \mathrm{C}$; IR ( $v$, $\mathrm{cm}^{-1}$ ): 3368, 3132, 3065, 2908, 1662, 1654, 1597, 1496, 1442, 1321; ${ }^{1} \mathrm{H}-\mathrm{NMR}$ (400 MHz, $\left.\mathrm{CDCl}_{3}\right) \delta$ (ppm): 10.23-10.21 ( $m, 2 \mathrm{H}, \mathrm{NH}), 7.99-7.97(m$, 1H, Ar-H), 7.69-7.67 ( $m, 2 \mathrm{H}$, Ar-H), 7.46-7.44 $(m, 1 \mathrm{H}, \mathrm{Ar}-\mathrm{H}), 7.30-7.22(m, 2 \mathrm{H}, \mathrm{Ar}-\mathrm{H}), 6.64$ $6.63(m, 1 \mathrm{H}, \mathrm{Ar}-\mathrm{H}), 6.36-6.35(m, 1 \mathrm{H}, \mathrm{Ar}-\mathrm{H})$, 4.29-4.27 ( $m, 2 \mathrm{H}, C H-\mathrm{NH}), 3.14-3.13(m, 2 \mathrm{H}$, $\mathrm{CH}_{2}-\mathrm{OH}$ ), 2.90-2.87 ( $\left.\mathrm{m}, 2 \mathrm{H}, \mathrm{CH}_{2}-\mathrm{OH}\right), 2.60-2.59$ $(m, 2 \mathrm{H}, \mathrm{CH}), 2.12\left(s, 3 \mathrm{H}, \mathrm{CH}_{3}\right), 1.54\left(s, 3 \mathrm{H}, \mathrm{CH}_{3}\right)$, $1.25\left(s, 12 \mathrm{H}, 4 \times \mathrm{CH}_{3}\right) ;{ }^{13} \mathrm{C}-\mathrm{NMR}(100 \mathrm{MHz}$, 
$\left.\mathrm{CDCl}_{3}\right) \delta(\mathrm{ppm}): 168.2,164.3,141.4,140.2,138.1$, $136.4,135.3,133.8,131.5,130.9,129.7,129.2$, $128.5,126.0,121.3,112.0,62.3,61.6,53.2,32.5$, 22.1, 18.1, 16.2. Anal. Calcd. for $\mathrm{C}_{29} \mathrm{H}_{38} \mathrm{~N}_{4} \mathrm{O}_{4}$ : C, 68.75; H, 7.56; N, 11.06. Found: C, 68.96; H, 7.52; $\mathrm{N}$, 11.01. (+)ESI-HRMS $\mathrm{m} / \mathrm{z}$ : calculated for $\left[\mathrm{C}_{33} \mathrm{H}_{29} \mathrm{~N}_{3} \mathrm{O}_{3}+\mathrm{H}^{+}\right]$: 507.2966; observed 507.2971. HPLC analysis: Chiral OD, $n$-hexane/ isopropyl

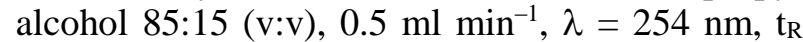
$($ minor $)=15.12 \mathrm{~min}, \mathrm{t}_{\mathrm{R}}($ major $)=17.53 \mathrm{~min}$.

\section{1-(2,5-Dimethylphenyl)- $N^{3}, N^{4}$-bis $(R)$-1- hydroxybutan-2-yl)-5-phenyl-1H-pyrazole- 3,4-dicarboxamide (10c)}

Compound 10c was prepared according to general procedure $(0.26 \mathrm{~g}, 90 \%)$. Mp: $249-251^{\circ} \mathrm{C}$; IR $(\mathrm{v}$, $\left.\mathrm{cm}^{-1}\right): 3318,3115,3064,2912,1664,1658,1596$, 1497, 1444, 1315; ${ }^{1} \mathrm{H}-\mathrm{NMR}$ (400 MHz, $\left.\mathrm{CDCl}_{3}\right) \delta$ (ppm): 10.64-10.61 (m, 2H, NH), 7.99-7.98 ( $m$, 1H, Ar-H), 7.67-7.63 (m, 2H, Ar-H), 7.34-7.22 (m, $3 \mathrm{H}, \mathrm{Ar}-\mathrm{H}), 6.86-6.85(\mathrm{~m}, 1 \mathrm{H}, \mathrm{Ar}-\mathrm{H}), 6.39-6.37(\mathrm{~m}$, 1H, Ar-H), 4.62-4.61 (m, 2H, CH-N), 3.75-3.73 (m, $\left.2 \mathrm{H}, \mathrm{CH}_{2}-\mathrm{OH}\right), 3.37-3.35\left(\mathrm{~m}, 2 \mathrm{H}, \mathrm{CH}_{2}-\mathrm{OH}\right), 2.60$ $2.57(m, 2 \mathrm{H}, \mathrm{CH}), 2.16\left(s, 3 \mathrm{H}, \mathrm{CH}_{3}\right), 1.91(m, 2 \mathrm{H}$, $\left.\mathrm{CH}_{2}-\mathrm{CH}_{3}\right), 1.59\left(s, 3 \mathrm{H}, \mathrm{CH}_{3}\right), 1.41\left(s, 6 \mathrm{H}, 2 \times \mathrm{CH}_{3}\right)$; ${ }^{13} \mathrm{C}-\mathrm{NMR}\left(100 \mathrm{MHz}, \mathrm{CDCl}_{3}\right) \delta$ (ppm): 170.0, 163.8, $143.6,138.1,136.0,133.2,131.3,130.4,129.9$, 129.6, 129.1, 128.8, 128.2, 121.0, 110.1, 58.4, 56.1, 52.3, 34.3, 24.5, 22.1, 19.0, 17.3, 15.0, 13.1. Anal. Calcd. for $\mathrm{C}_{29} \mathrm{H}_{38} \mathrm{~N}_{4} \mathrm{O}_{4}$ : C, 67.76; H, 7.16; N, 11.71 . Found: C, 68.76; H, 7.51; N, 11.77. (+)ESI-HRMS $m / z$ : calculated for $\left[\mathrm{C}_{33} \mathrm{H}_{29} \mathrm{~N}_{3} \mathrm{O}_{3}+\mathrm{H}^{+}\right]$: 479.2653; observed 479.2648. HPLC analysis: Chiral OD, $n$ -

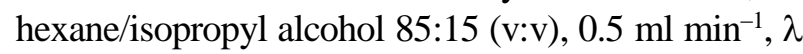
$=254 \mathrm{~nm}, \mathrm{t}_{\mathrm{R}}($ minor $)=15.23 \mathrm{~min}, \mathrm{t}_{\mathrm{R}}($ major $)=17.51$ min.

\subsection{Enantiomeric recognition experiments}

\section{General procedure for asymmetric Henry reaction}

The compounds 4a-c, 5a-c,7a-c or 10a-c $(0.01 \mathrm{mmol})$ and $\mathrm{CuCl}(0.01 \mathrm{mmol})$ were added to absolute ethanol $(0.5 \mathrm{ml})$. After the reaction mixture's color turned to green, $p$-nitro benzaldehyde $(0.1 \mathrm{mmol})$ was added, and then nitromethane $(0.1$ mmol) was added slowly using a syringe. The reaction mixture was stirred at $35^{\circ} \mathrm{C}$ for $24 \mathrm{~h}$. The reaction was monitored by TLC. When reaction was completed, $\mathrm{HCl}(1 \mathrm{M}, 3 \mathrm{ml})$ and $\mathrm{CH}_{2} \mathrm{Cl}_{2}(1 \mathrm{ml})$ were added to the mixture. Then extraction was done with $\mathrm{CH}_{2} \mathrm{Cl}_{2}(3 \times 10 \mathrm{ml})$. Organic phases were combined and dried over $\mathrm{MgSO}_{4}$. Solvent was evaporated in vacuum, and the product was washed with diethyl ether. It was purified by petroleum ether/ethyl acetate $\left(70: 30, R_{f}=0.36\right)$.

\subsection{Computational method}

The three-dimensional ground state $\left(\mathrm{S}_{0}\right)$ geometries of all compounds were optimized in the gas phase without any symmetry restrictions using DFT [23] implemented hybrid functional B3LYP with the Gaussian 09W [24] software package. B3LYP is composed of Becke's three parameter exchange functional (B3) [25] and the non-local correlation functional by Lee, Yang and Parr (LYP) [26]. In order to find the molecular structures with minimum energy, conformational analysis was performed with the B3LYP/6-311++G(d,p) method and basis set.

The vibrational analyses were carried out using the same basis set employed in the corresponding geometry optimizations. The frequency analysis did not yield any imaginary frequencies, indicating that the optimized structure of the molecule corresponds to at least a local minimum on the potential energy surface. The normal mode analysis was performed for $3 \mathrm{~N}-6$ vibrational degrees of freedom, with $\mathrm{N}$ being the number of atoms in the molecule.

\section{RESULTS AND DISCUSSION}

\subsection{Chemistry}

Initially, pyrazole-3-carboxylic acid $\mathbf{1}$ was synthesized according to previously reported procedure [22], and the corresponding acid chloride $\mathbf{2}$ was obtained by heating pyrazole-3-carboxylic acid with an excess of $\mathrm{SOCl}_{2}[21,22]$. When compound 2 was reacted with appropriate chiral amino alcohols at room temperature, both $-\mathrm{NH}_{2}$ groups and $-\mathrm{OH}$ groups of the amino alcohols, which have strong nucleophilic properties, attacked the acyl carbons. As a result of that reaction, chiral pyrazole-3-carboxamides 4a-c and different novel chiral pyrazole-3-carboxylates 5a-c were obtained in 88-12\% yield (Scheme 1). On the other hand, we used pyrazole-3-carboxylate $\mathbf{3}$ as starting compound to obtain pyrazole-3-carboxamide 4a-c derivatives as the sole products. All of the synthesized compounds were characterized by spectroscopic methods (see experimental part).

The IR spectrum of chiral pyrazole-3-carboxylate 5a-c showed absorption bands at 3125$3142 \mathrm{~cm}^{-1}$ corresponding to $-\mathrm{NH}_{2}$ groups and at 1710-1708 $\mathrm{cm}^{-1}$ corresponding to ester carbonyl groups. The ${ }^{1} \mathrm{HNMR}$ spectra of 5a-c revealed signals of $-\mathrm{CH}=,-\mathrm{CH}_{2}$ and $-\mathrm{NH}-$ protons at 3.4-2.8, 4.4-3.7, $1.9-1.5 \mathrm{ppm}$, respectively. Their ${ }^{13} \mathrm{CNMR}$ spectra revealed the carbonyl signals of benzoyl groups at 194.6-191.4 ppm and the carbonyl of ester groups at 163.6-158.3 ppm. 


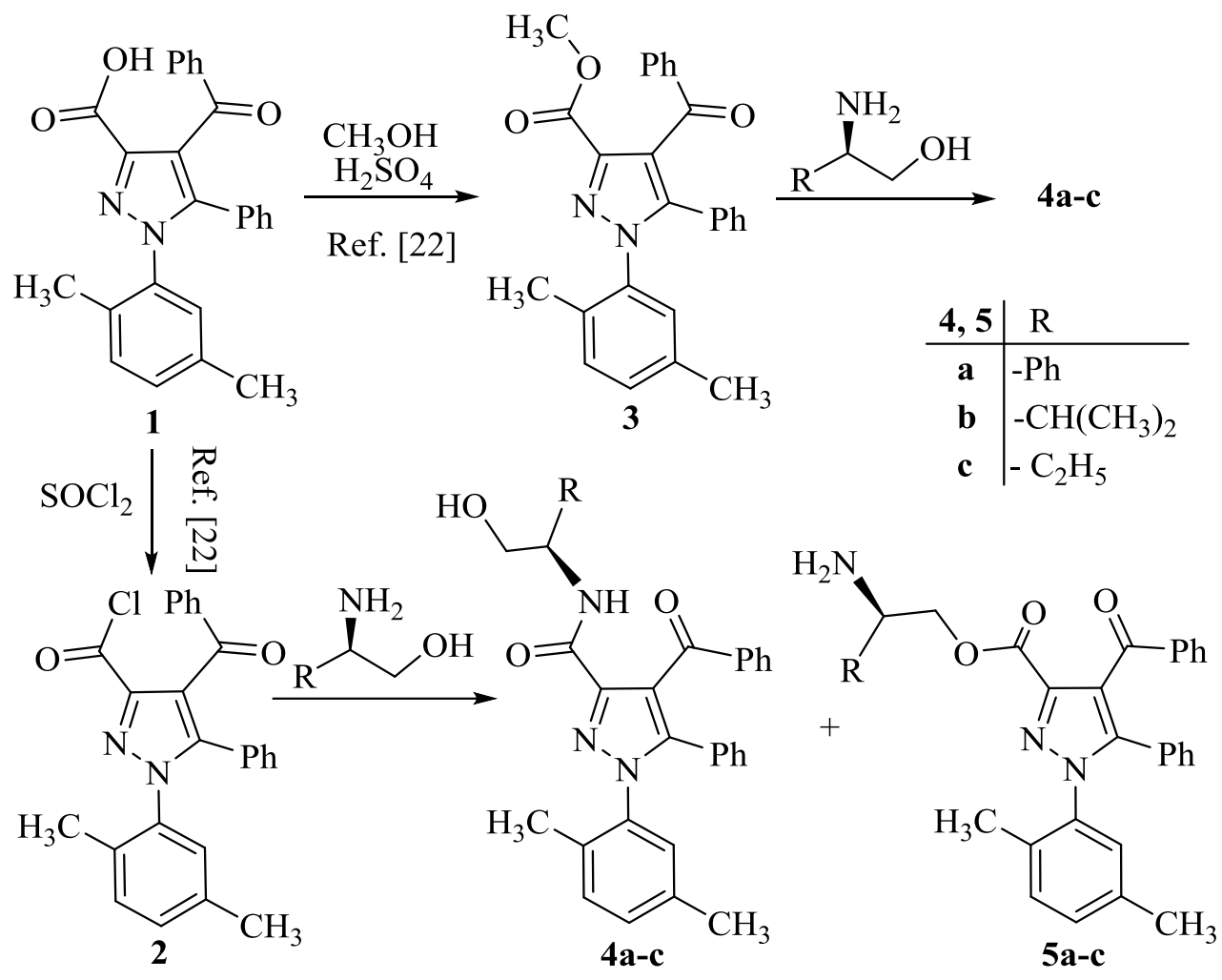

Scheme 1. Synthesis of chiral pyrazole-centered carboxamide 4a-c and ester 5a-c derivatives

To afford the synthesis of chiral molecules, we prepared a series of chiral pyrazolo-thiourea derivatives (7a-c) by the reactions of 4-benzoyl-1(2,5-dimethylphenyl)-5-phenyl-1 $H$-pyrazole-3-carbonyl-isothiocyanate (6) and the chiral amino alcohols (Scheme 2) [21]. The structures of the synthesized chiral molecules were confirmed by spec- troscopic methods and by comparing physical properties such as melting points to the data in our previous work [22]. Finally, we investigated, for the first time, the synthesized compounds that possess tetrasubstituted pyrazole derivatives for their catalytic properties in asymmetric synthesis reactions.<smiles>[R]C(N)C(O)Cn1nc(C(=O)N=C=S)c(C(=O)c2ccccc2)c1-c1cc(C)ccc1C</smiles><smiles>[R]C(CO)NC(=S)NC(=O)c1nn(-c2cc(C)ccc2C)c(-c2ccccc2)c1C(=O)c1ccccc1</smiles>

Scheme 2. Synthesis of chiral pyrazolo-thiourea derivatives, 7a-c

Moreover, the bidentate chiral compounds have shown better catalytic activity in asymmetric synthesis, according to the results of a literature search [27-29]. Therefore, to obtain bidentate chiral compounds, the pyrazole-3,4-dicarboxyester 9 was synthesized by an esterification reaction of the starting compound $\mathbf{8}$ according to our data reported previously [22]. Afterwards, compound 9 was converted into the bidentate chiral derivatives 10a-c by using chiral amino alcohols (Scheme 3). 
<smiles>[R]C(CO)NC(=O)c1nn(-c2cc(C)ccc2C)c(-c2ccccc2)c1C(=O)NC([R])CO</smiles>

Scheme 3. Synthesis of chiral pyrazole-bis(carboxamide) derivatives 10a-c

Structure confirmations of chiral pyrazole3,4-dicarboxamide derivatives 10a-c are mainly based on their ${ }^{1} \mathrm{H}$ and ${ }^{13} \mathrm{CNMR}$ spectral data. Signals of amide, methine and methylene carbon atoms are assigned at 170.0-163.8, 53.2-46.1, 62.3$53.5 \mathrm{ppm}$, respectively. Signals observed at 10.69.6, 4.8-4.2, 3.7-3.1 ppm in the ${ }^{1} \mathrm{HNMR}$ spectra of 10a-c point to signals of $-\mathrm{NH},-\mathrm{CH}=$, and $-\mathrm{CH}_{2}-$ protons, respectively.

\subsection{Catalytic studies}

Eventually, all of the synthesized compounds (4a-c, 5a-c, 7a-c, 10a-c) having chiral thioureide, carboxamide, ester and dicarboxamide groups were evaluated for enantiomeric recognition properties in the Henry reactions. Chiral ligands were used as catalysts in the reaction between nitromethane and $p$-nitrobenzaldehyde (Table 1, entries 1-12). The reaction was performed in $\mathrm{EtOH}$ at $35^{\circ} \mathrm{C}$ with $\mathrm{CuCl}$.

$\mathrm{T}$ a ble 1

The Henry reaction between nitromethane and p-nitrobenzaldehyde ${ }^{a}$<smiles>CCO[N+](=O)[O-]</smiles>

\begin{tabular}{cccccccc}
\hline \hline Entry & Catalyst & Solvent & Lewis acid & $t$ (hour) & Temp. $\left({ }^{\circ} \mathrm{C}\right)$ & Yield $^{\mathrm{b}}(\%)$ & $\mathrm{Ee}^{\mathrm{c}}(\%)(R)$ \\
\hline 1 & $4 \mathrm{a}$ & $\mathrm{EtOH}$ & $\mathrm{CuCl}$ & 24 & 35 & 76 & 14 \\
2 & $4 \mathrm{~b}$ & $\mathrm{EtOH}$ & $\mathrm{CuCl}$ & 24 & 35 & 78 & 11 \\
3 & $4 \mathrm{c}$ & $\mathrm{EtOH}$ & $\mathrm{CuCl}$ & 24 & 35 & 72 & 7 \\
4 & $5 \mathrm{a}$ & $\mathrm{EtOH}$ & $\mathrm{CuCl}$ & 24 & 35 & 12 & $<5$ \\
5 & $5 \mathrm{~b}$ & $\mathrm{EtOH}$ & $\mathrm{CuCl}$ & 24 & 35 & 15 & $<5$ \\
6 & $5 \mathrm{c}$ & $\mathrm{EtOH}$ & $\mathrm{CuCl}$ & 24 & 35 & 15 & $<5$ \\
7 & $7 \mathrm{a}$ & $\mathrm{EtOH}$ & $\mathrm{CuCl}$ & 24 & 35 & 70 & 32 \\
8 & $7 \mathrm{~b}$ & $\mathrm{EtOH}$ & $\mathrm{CuCl}$ & 24 & 35 & 65 & 16 \\
9 & $7 \mathrm{c}$ & $\mathrm{EtOH}$ & $\mathrm{CuCl}$ & 24 & 35 & 65 & 16 \\
10 & $10 \mathrm{a}$ & $\mathrm{EtOH}$ & $\mathrm{CuCl}$ & 24 & 35 & 86 & 54 \\
11 & $10 \mathrm{~b}$ & $\mathrm{EtOH}$ & $\mathrm{CuCl}$ & 24 & 35 & 88 & 36 \\
12 & $10 \mathrm{c}$ & $\mathrm{EtOH}$ & $\mathrm{CuCl}$ & 24 & 35 & 90 & 32 \\
\hline \hline
\end{tabular}

a. Reaction conditions: aldehyde $(0.1 \mathrm{mmol})$, nitromethane $(0.1 \mathrm{mmol})$, catalyst $(0.01 \mathrm{mmol})$

b. Isolated yield

c. Enantiomer excesses were determined by chiral HPLC and absolute configurations $(R$-isomer or $S$-isomer) were assigned by comparison of optical rotation data and retention time with the literature data [30-32]. 
Compound 10a was selected to optimize reaction conditions in which temperature, solvent, Lewis acid and reaction time (Table 2, entries 113) were investigated. Decreases in the temperature resulted in decreasing both chemical yields and enantioselectivities (Table 2, entries 2, 11 and 12), and when the reaction was performed at $35^{\circ} \mathrm{C}$, the aldol product was obtained in $54 \%$ ee. The various solvents evaluated for the asymmetric Hen- ry reactions between nitromethane and $p$-nitrobenzaldehyde are shown in Table 2 (entries 1-4). When the ligand 10a was used for the nitro aldol reaction under similar conditions, poor yields were obtained both in DMF and THF, and they gave 10, 12 enantioselectivity (Table 2 , entries 1,4 ). Ethanol was found to be the best solvent for this reaction (Table 2, entry 2).

Table 2

Screening of reaction parameters for Henry reaction ${ }^{a}$

\begin{tabular}{cccccccc}
\hline \hline Entry & Catalyst & Solvent & Lewis Acid & $\mathrm{t}$ (hour) & Temp. $\left({ }^{\circ} \mathrm{C}\right)$ & Yield $^{\mathrm{b}}(\%)$ & Ee $^{\mathrm{c}}(\%)(\mathrm{R})$ \\
\hline 1 & $10 \mathrm{a}$ & $\mathrm{DMF}$ & $\mathrm{CuCl}$ & 24 & 35 & 42 & 12 \\
2 & $10 \mathrm{a}$ & $\mathrm{EtOH}$ & $\mathrm{CuCl}$ & 24 & 35 & 86 & 54 \\
3 & $10 \mathrm{a}$ & $\mathrm{CH}_{2} \mathrm{Cl}_{2}$ & $\mathrm{CuCl}$ & 24 & 35 & 72 & 16 \\
4 & $10 \mathrm{a}$ & $\mathrm{THF}$ & $\mathrm{CuCl}$ & 24 & 35 & 54 & 10 \\
5 & $10 \mathrm{a}$ & $\mathrm{EtOH}$ & $\mathrm{Cu}(\mathrm{OAc})_{2}$ & 24 & 35 & 80 & 20 \\
6 & $10 \mathrm{a}$ & $\mathrm{EtOH}$ & $\mathrm{CuI}$ & 24 & 35 & 82 & 24 \\
7 & $10 \mathrm{a}$ & $\mathrm{EtOH}$ & $\mathrm{CuBr}$ & 24 & 35 & 40 & $<5$ \\
8 & $10 \mathrm{a}$ & $\mathrm{EtOH}$ & $\mathrm{CuCl}$ & 12 & 35 & 80 & $<5$ \\
9 & $10 \mathrm{a}$ & $\mathrm{EtOH}$ & $\mathrm{CuCl}$ & 36 & 35 & 64 & 20 \\
10 & $10 \mathrm{a}$ & $\mathrm{EtOH}$ & $\mathrm{CuCl}$ & 48 & 35 & 50 & $<5$ \\
11 & $10 \mathrm{a}$ & $\mathrm{EtOH}$ & $\mathrm{CuCl}$ & 24 & 0 & 64 & $<5$ \\
12 & $10 \mathrm{a}$ & $\mathrm{EtOH}$ & $\mathrm{CuCl}$ & 24 & 20 & 70 & 22 \\
13 & $10 \mathrm{a}$ & $\mathrm{EtOH}$ & $\mathrm{CuCl}$ & 24 & 50 & 84 & $<5$ \\
\hline \hline
\end{tabular}

a. Reaction conditions: aldehyde $(0.1 \mathrm{mmol})$, nitromethane $(0.1 \mathrm{mmol})$, catalyst $(0.01 \mathrm{mmol})$

b. Isolated yield

c. Enantiomer excesses were determined by chiral HPLC and absolute configurations ( $R$-isomer or $S$-isomer) were assigned by comparison of optical rotation data and retention time with the literature data [30-32].

The Lewis acids used in this study are shown in Table 2 (entries 2, 5-7). $\mathrm{CuCl}$, $\mathrm{Cu}(\mathrm{OAc})_{2}, \mathrm{CuI}$ and $\mathrm{CuBr}$ were chosen for the nitro aldol reactions. $\mathrm{CuCl}$ was found to give the best results for both chemical yield and enantioselectivity values for this catalyst reaction (Table 2, entry 2). $\mathrm{CuBr}$ did not show good selectivity, and the reaction in $\mathrm{CuBr}$ gave enantiomeric excess down to $5 \%$ (Table 2, entry 7). In addition, the optimal time for asymmetric Henry reactions was investigated (Table 2, entries 2, 8-10). For example, when the reaction was performed for 12 hours, ee $<5 \%$ and $80 \%$ yield were obtained (Table 2, entry 8 ). When the reaction was performed for 24 hours, a high yield ( $86 \%$ ) and enantioselectivity (54\%) were obtained (Table 2, entry 2). However, if the reaction was performed for 36 hours, a high yield (64\%) but low enantioselectivity (20\%) were obtained (Table 2, entry 9). Moreover, when the reaction lasted 48 hours, a moderate yield (50 $\%)$ and low enantioselectivity $(<5 \%)$ were obtained (Table 2, entry 10). In summary, appropriate temperature, solvent, Lewis acid, and reaction time were indicated by several tests, and the optimized conditions are shown in Table 2, entries 1-13.

Under optimized conditions, ligand 10a showed more enantioselectivity than the other ligands (Table 1). This may be due to the fact that the phenyl moieties interacted with $p$-nitrobenzaldehyde and the aromatic ring provided steric hindrance during the reaction. It was difficult for the substrate to combine with copper complex. The catalytic mechanism of compound $\mathbf{1 0 a}$, proposed based on literature [32], is given in the Figure 2. 


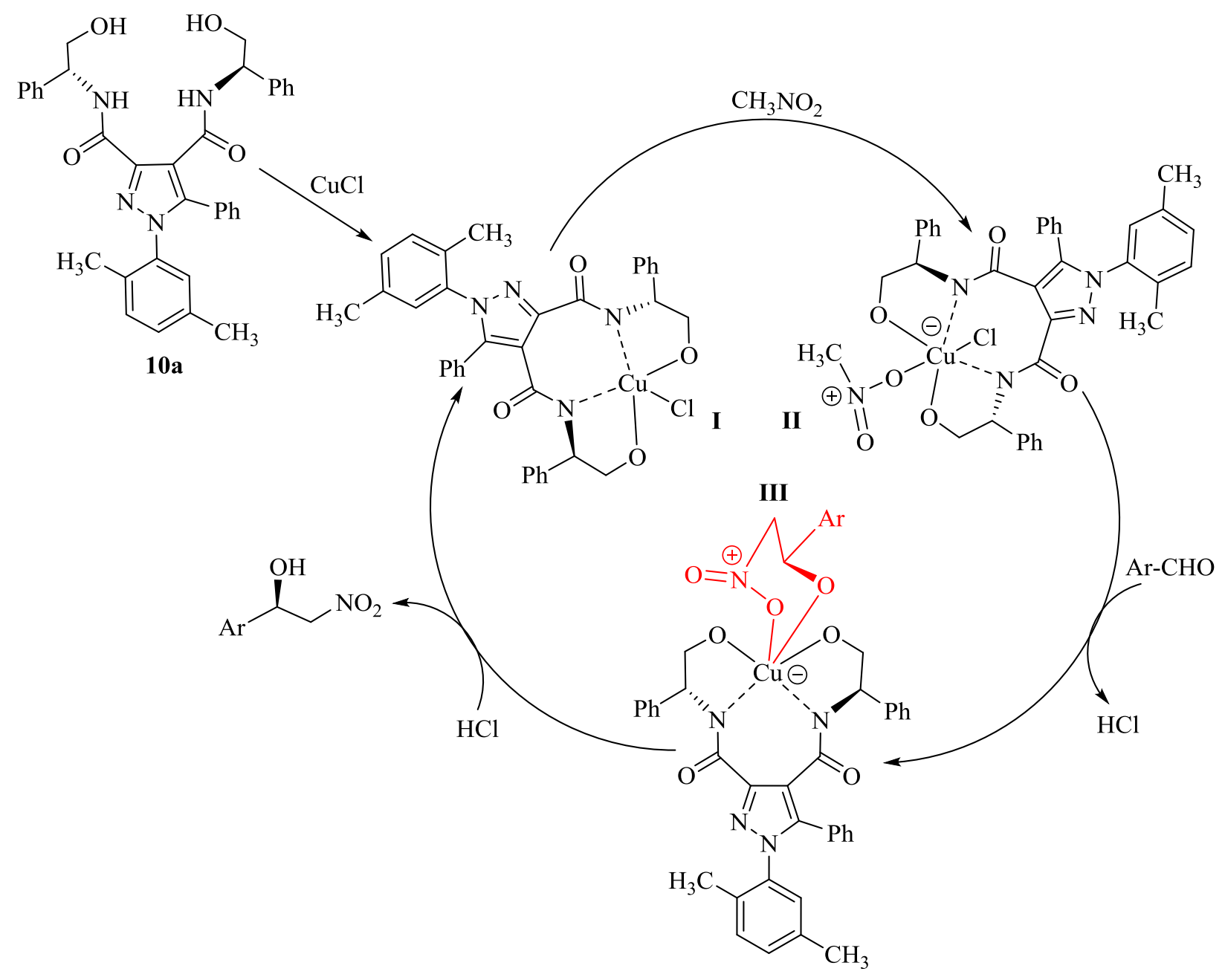

Fig. 2. Proposed catalytic mechanism of asymmetric nitro aldol reaction for 10a

\subsection{Computational studies}

Optimized geometry structures of the compounds obtained from B3LYP/6-31++G(d,p) computations are given in Figure 3. Hydrogens are subtracted from the systems for clarity. According to the data in Table 1, compounds 10a-c showed better yields and enantioselectivity over the others, 10a being the best. The geometry optimized structures indicate that the coordination cavity for a $\mathrm{Cu}^{+}$ cation can be observed to be better for these systems than the others. This may account for the increased yield and selectivity. 3D electrostatic potential maps for the present structures are shown in Figure 4. Red areas indicate more negative electron development. Structures $4 \mathbf{a}$ and 10a possess the most suitable cavities for complexation with a copper cation in the intermediate step, which is reflected by the high yields with these ligands.
The Highest Occupied Molecular Orbital (HOMO) implies the outermost filled orbital, and it behaves as an electron donor. On the other hand, the Lowest Unoccupied Molecular Orbital (LUMO) can be considered to be the unfilled orbital with lowest energy, and it behaves as an electron acceptor. HOMO and LUMO are also called the frontier molecule orbitals (FMOs). The energy gap between FMOs gives information about the chemical stability of a molecule, and it is an important parameter in terms of electronic transport properties. In Figure 5, a part of the orbital energies (in $\mathrm{eV}$ ) and three-dimensional HOMO and LUMO energy schemes for 4a and 10a are given. HOMO and LUMO orbitals are located around the carbonyl moiety for 4a. However, they are found to be on the pyrazole moiety in 10a. The computed HOMO and LUMO energies are -6.16 and $-2.15 \mathrm{eV}$ for 4a, and -6.48 and $-1.18 \mathrm{eV}$ for $\mathbf{1 0 a}$. 


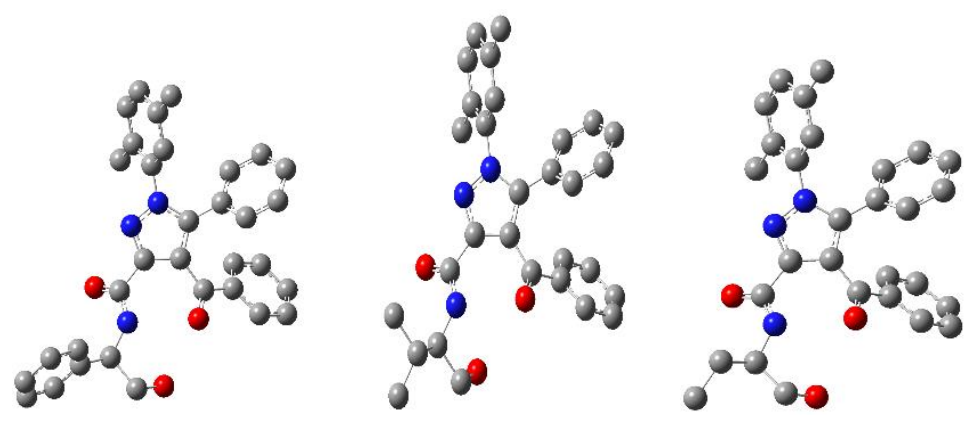

$4 \mathbf{a}$

$4 \mathbf{b}$

$4 c$

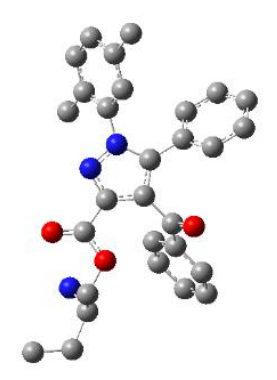

$5 \mathbf{a}$

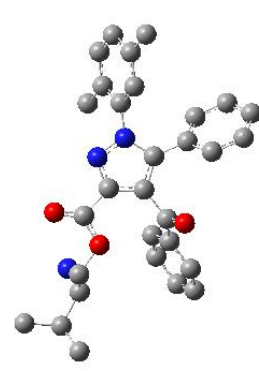

$\mathbf{5 b}$

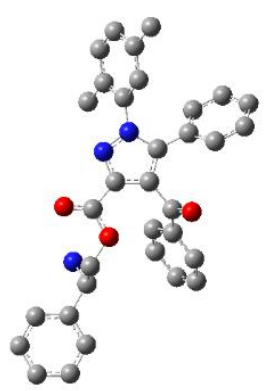

$5 c$

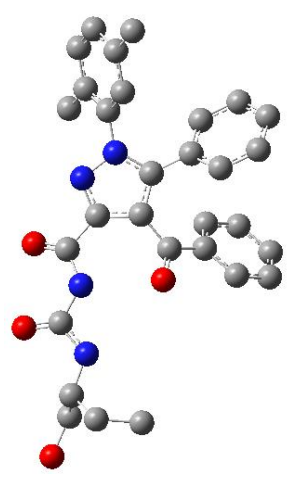

$7 \mathbf{a}$

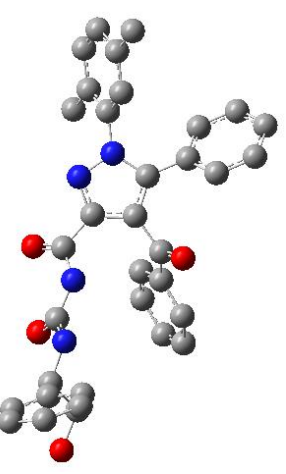

$7 \mathbf{b}$

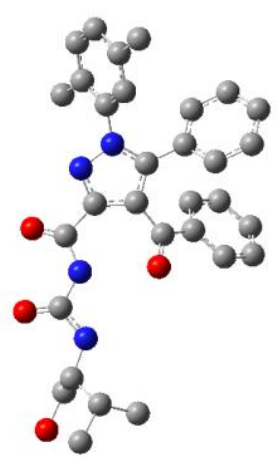

7c

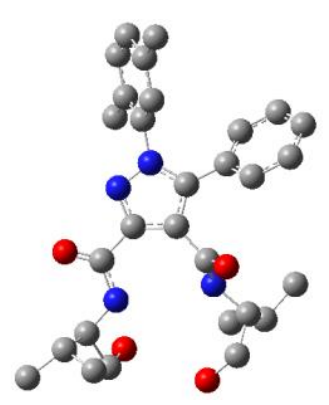

$10 \mathbf{a}$

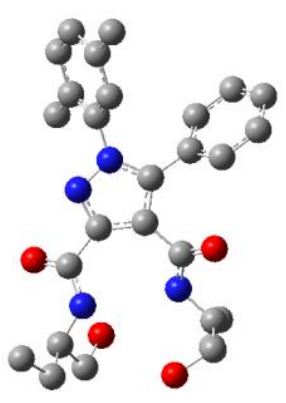

$10 b$

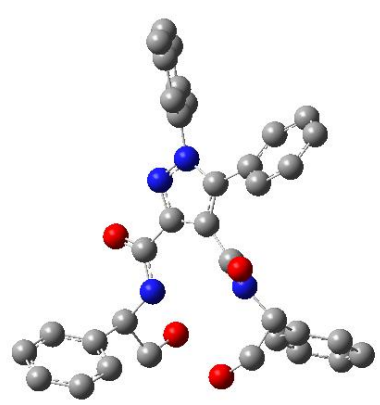

10c

Fig. 3. Geometry optimized structures of the novel compounds 


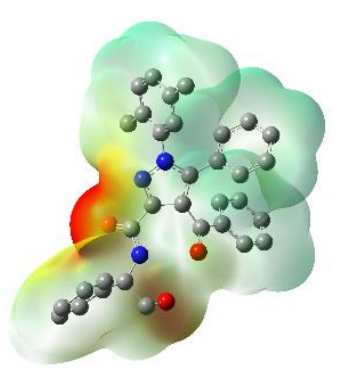

$4 a$

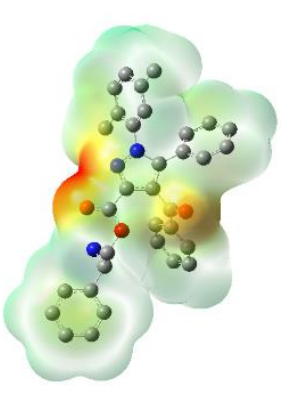

$5 \mathbf{a}$

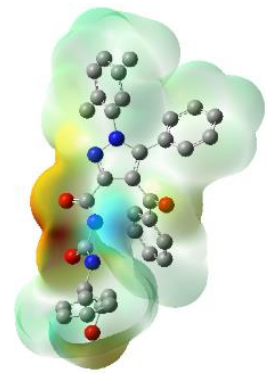

$7 \mathbf{a}$

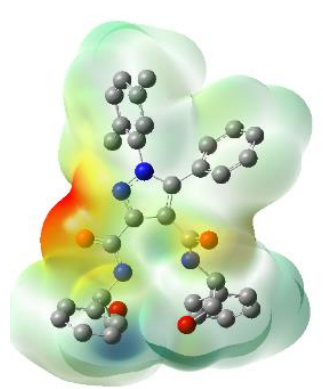

10a

Fig. 4. 3D electrostatic potential maps of the present compounds

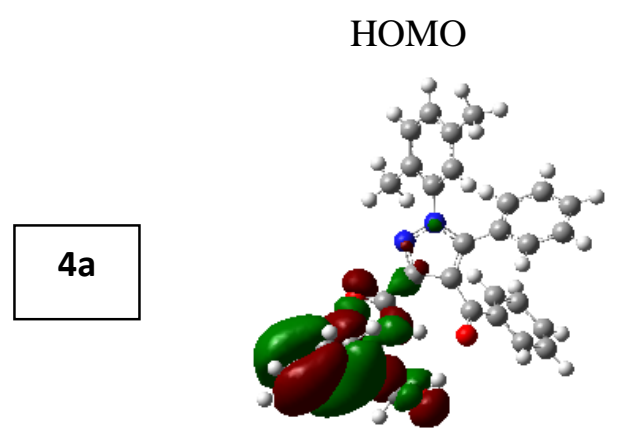

$10 \mathrm{a}$

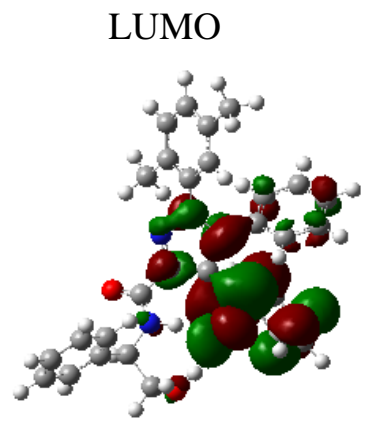

$-2.15 \mathrm{eV}$

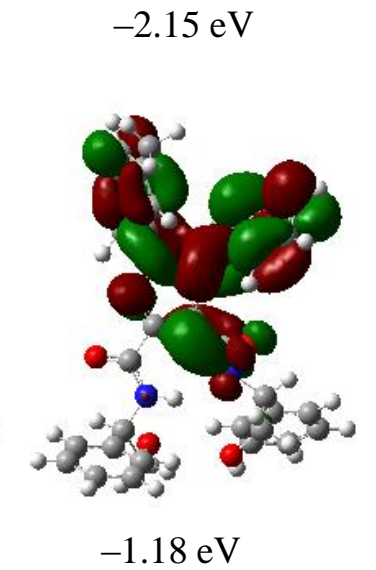

$-1.18 \mathrm{eV}$
$-6.16 \mathrm{eV}$

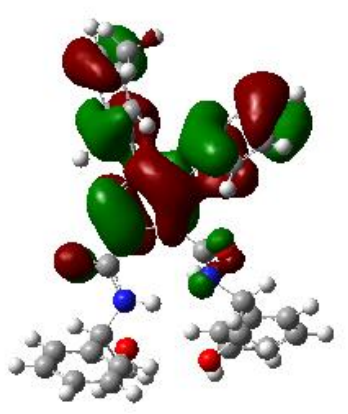

$-6.48 \mathrm{eV}$
Fig. 5. FMO schemes and energies of $4 \mathbf{a}$ and 10a

\section{CONCLUSIONS}

In conclusion, several kinds of organocatalysts with a tetra substituted pyrazole skeleton have been efficiently employed for asymmetric aldol reactions in which the pyrazoles bear chiral thioureide, carboxamide, ester and dicarboxamide groups. The bidentate chiral compound 10a has shown the best catalytic activity. In addition, these synthesized catalysts may be applied to the other asymmetric synthesis reactions. For this reason, the methods developed for the synthesis of these compounds are becoming more important. Apart from the synthetic procedures, some computations have been performed to gain information about the structural and electronic properties of these compounds.

Acknowledgements. The authors thank to the Management Unit of Scientific Research Projects of Mus Alparslan University for financial support under Project MSÜ14EMF-G05.

\section{REFERENCES}

[1] W. Ma, H. Kuang, L. Xu, L. Ding, C. Xu, L. Wang, N. A. Kotov, Attomolar DNA detection with chiral nanorod assemblies, Nat. Commun. 4, 2689 (2013). DOI: doi.org/10.1038/ncomms3689

[2] B. Wei, N. Liu, J. Zhang, X. Ou, R. Duan, Z. Yang, F. Xia, Regulation of DNA self-assembly and DNA hy- 
bridization by chiral molecules with corresponding biosensor applications, Analytic. Chem. 87, 2058-2062 (2015). DOI: doi.org/10.1021/ac504797e

[3] M. Liu, L. Zhang, T. Wang, Supramolecular chirality in self-assembled systems, Chem. Rev. 115, 7304-7397 (2015). DOI: doi.org/10.1021/cr500671p

[4] K. Ohmatsu, T. Ooi, Design of supramolecular chiral ligands for asymmetric metal catalysis, Tetrahedron Lett. 56, 2043-2348 (2015).

DOI: doi.org/10.1016/j.tetlet.2015.02.096

[5] A. G. Maldonado, J. P. Doucet, M. Petitjean, B. T. Fan, Molecular similarity and diversity in chemoinformatics: from theory to applications. Molec. Divers. 10, 39-79 (2006). DOI: doi.org/10.1007/s11030-006-8697-1

[6] J. M. Woodley, New opportunities for biocatalysis: making pharmaceutical processes greener, Trends Biotechnol. 26, 321-327 (2008).

DOI: doi.org/10.1016/j.tibtech.2008.03.004

[7] S. Saeed, N. Rashid, P. G. Jones, R. Hussain, Synthesis, characterization and biological evaluation of some thiourea derivatives bearing benzothiazole moiety as potential antimicrobial and anticancer agents, Eur. J. Med. Chem. 45, 1323-1331 (2010). DOI: doi.org/10.1016/j.ejmech.2009.12.016

[8] R. A. Sheldon, Chirotechnology: Industrial synthesis of optically active compounds, CRC Press (1993). DOI: doi.org/10.1002/chir.530060109

[9] L. Jiang, H. T. Zheng, T. Y. Liu, L. Yue, Y. C. Chen, Asymmetric direct vinylogous carbon-carbon bond formation catalyzed by bifunctional organocatalysts, Tetrahedron, 63, 5123-5128 (2007).

DOI: doi.org/10.1016/j.tet.2007.04.011

[10] N. Kumarswamyreddy, V. Kesavan, Enantioselective synthesis of dihydrospiro[indoline-3,4'-pyrano[2,3c]pyrazole] derivatives via Michael/Hemiketalization Reaction, Org. Lett. 18, 1354-1357 (2016). DOI: doi.org/10.1021/acs.orglett.6b00287

[11] P. Chauhan, S. Mahajan, D. Enders, Asymmetric synthesis of pyrazoles and pyrazolones employing the reactivity of pyrazolin-5-one derivatives, Chem. Commun. 51, 1289012907 (2015). DOI: doi.org/10.1039/C5CC04930J

[12] L. A. Chen, W. Xu, B. Huang, J. Ma, L. Wang, J. Xi, E. Meggers, Asymmetric Catalysis with an Inert Chiral-atMetal Iridium Complex, J. Am. Chem. Soc. 135, 1059810601 (2013). DOI: doi.org/10.1021/ja403777k

[13] H.-M. Zhang, H. Lv, S. Ye, N-Heterocyclic carbenecatalyzed highly enantioselective synthesis of substituted dihydropyranopyrazolones, Org. Biomol. Chem. 11, 6255-6257 (2013). DOI: doi.org/10.1039/C4OB02330G

[14] C. Vila, F. I. Amr, G. Blay, M. C. Muñoz, J. R. Pedro, Organocatalytic enantioselective synthesis of pyrazoles bearing a quaternary stereocenter, Chem. Asia J. 11, 1532-1536 (2016).

DOI: doi.org/10.1002/asia.201600325

[15] J. Xie, X. Y. Xing, F. Sha, Z. Y. Wu, X. Y. Wu, Enantioselective synthesis of spiro[indoline-3,4'-pyrano[2,3c]pyrazole] derivatives via an organocatalytic asymmetric Michael/cyclization cascade reaction, Org. Biomol. Chem. 14, 8346-8355 (2016). DOI: doi.org/10.1039/C6OB01256F
[16] D. M. Bailey, P. E. Hansen, A. G. Hlavac, E. R. Baizman, J. Pear, A. F. DeFelice, M. E. Feigensonf, 3, 4Diphenyl-1H-pyrazole-1-propanamine antidepressants, J. Med. Chem. 28, 256-260 (1985). DOI: doi.org/10.1021/jm00380a020

[17] T. L. Siddall, D. G. Ouse, Z. L. Benko, G. M. Garvin, J. L. Jackson, J. M. McQuiston, M. J. Ricks, T. D. Thibault, J. A. Turner, J. C. VanHeertum, M. R. Weimer, Synthesis and herbicidal activity of phenyl-substituted benzoylpyrazoles, Pest Manag Sci 58, 1175-1186 (2002). DOI: doi.org/10.1002/ps.588

[18] H. Tahermansouri, E. Biazar, Functionalization of carboxylated multi-wall carbon nanotubes with 3,5diphenyl pyrazole and an investigation of their toxicity, New Carbon Mater 28, 199-207 (2013). DOI: doi.org/10.1016/S1872-5805(13)60077-3

[19] N. K. Terrett, A. S. Bell, D. Brown, P. Ellis, Sildenafil $\left(\right.$ Viagra $\left.^{\mathrm{TM}}\right)$, a potent and selective inhibitor of type 5 CGMP phosphodiesterase with utility for the treatment of male erectile dysfunction, Bioorg. Med. Chem. Let. 6, 1819-1824 (1996). DOI: doi.org/10.1016/0960-894X(96)00323-X

[20] V. Ramkumar, P. Kannan, Novel heterocyclic based blue and green emissive materials for opto-electronics. Opt Mater 46, 314-323 (2015). DOI: doi.org/10.1016/j.optmat.2015.04.038

[21] I. Bildirici, A. Cetin, N. Menges, Y. Alan, Synthesis and SAR studies of pyrazole-3-carboxamides and -thioureides including chiral moiety: Novel candidates as antibacterial agents, J. Serb. Chem. Soc. 83, 795-807 (2018). DOI: doi.org/10.2298/JSC170313029B

[22] A. Çetin, İ. Bildirici, A study on synthesis and antimicrobial activity of 4-acyl-pyrazoles. J. Saud. Chem. Soc. 22, 279-296 (2016). DOI: doi.org/10.1016/j.jscs.2016.05.008

[23] W. Kohn, L. J. Sham, Self-consistent equations including exchange and correlation effects, Phys. Rev. 140, 1133-1138 (1965). DOI: doi.org/10.1103/PhysRev.140.A1133

[24] M. J. Frisch, G. W. Trucks, H. B. Schlegel, G. E. Scuseria, M. A. Robb et al., Gaussian 09, Revision D.01. Wallingford CT, USA: Gaussian, Inc., 2013.

[25] A. D. Becke, Density-functional exchange-energy approximation with correct asymptotic behaviour, Phys. Rev. A 38, 3098-3100 (1988). DOI: doi.org/10.1103/PhysRevA.38.3098

[26] C. Lee, W. Yang, R. G. Parr, Development of the ColleSalvetti correlation energy formula into a functional of the electron density, Phys. Rev. B 37, 785-789 (1988). DOI: doi.org/10.1103/physrevb.37.785

[27] P. J. Guiry, C. P. Saunders, The development of bidentate P,N-ligands for asymmetric catalysis, Adv. Synth. Catal. 346, 497-537 (2004). DOI: doi.org/10.1002/adsc.200303138

[28] H. Willms, W. Frank, C. Ganter, Coordination chemistry and catalytic application of bidentate phosphaferrocenepyrazole and -imidazole based P,N-ligands, Organometallics 28, 3049-3058 (2009). DOI: doi.org/10.1021/om8012025 
[29] M. Bartok, Unexpected inversions in asymmetric reactions: reactions with chiral metal complexes, chiral organocatalysts, and heterogeneous chiral catalysts, Chem. Rev. 110, 1663-1705 (2009).

DOI: doi.org/10.1021/cr9002352

[30] M. Watanabe, K. Murata, T. Ikariya, Practical synthesis of optically active amino alcohols via asymmetric transfer hydrogenation of functionalized aromatic ketones, $J$.

Org. Chem. 67, 1712-1715 (2002).

DOI: doi.org/10.1021/jo011076w
[31] H. Sasai, T. Suzuki, S. Arai, T. Arai, M. Shibasaki, Basic character of rare earth metal alkoxides. Utilization in catalytic carbon-carbon bond-forming reactions and catalytic asymmetric nitro aldol reactions, J. Am. Chem. Soc. 114, 4418-4420 (1992). DOI: doi.org/10.1021/ja00037a068

[32] D. A. Evans, D. Seidel, M. Rueping, H. W. Lam, J. T. Shaw, C. W. Downey, A New copper acetatebis(oxazoline)-catalyzed, Enantioselective Henry reaction, J. Am. Chem. Soc. 125, 12692-12693 (2003). DOI: doi.org/10.1021/ja0373871 